\title{
Uniform hypothesis testing for finite-valued stationary processes
}

\author{
Daniil Ryabko *
}

\begin{abstract}
Given a discrete-valued sample $X_{1}, \ldots, X_{n}$ we wish to decide whether it was generated by a distribution belonging to a family $H_{0}$, or it was generated by a distribution belonging to a family $H_{1}$. In this work we assume that all distributions are stationary ergodic, and do not make any further assumptions (e.g. no independence or mixing rate assumptions). We would like to have a test whose probability of error (both Type I and Type II) is uniformly bounded. More precisely, we require that for each $\varepsilon$ there exist a sample size $n$ such that probability of error is upper-bounded by $\varepsilon$ for samples longer than $n$. We find some necessary and some sufficient conditions on $H_{0}$ and $H_{1}$ under which a consistent test (with this notion of consistency) exists. These conditions are topological, with respect to the topology of distributional distance.
\end{abstract}

\section{Introduction}

Given a sample $X_{1}, \ldots, X_{n}$ (where $X_{i}$ are from a finite alphabet $A$ ) which is known to be generated by a stationary ergodic process, we wish to decide whether it was generated by a distribution belonging to a family $H_{0}$, versus it was generated by a distribution belonging to a family $H_{1}$. Unlike most of the works on the subject, we do not assume that $X_{i}$ are i.i.d., but only make a much weaker assumption that the distribution generating the sample is stationary ergodic.

A test is a function that takes a sample and gives a binary (possibly incorrect) answer: either the sample was generated by a distribution from $H_{0}$ or by a distribution from $H_{1}$. An answer $i \in\{0,1\}$ is correct if the sample is generated by a distribution that belongs to $H_{i}$. Here we are concerned with characterizing those pairs of $H_{0}$ and $H_{1}$ for which consistent tests exist.

Consistency. In this work we consider the following notion of consistency. For two hypothesis $H_{0}$ and $H_{1}$, a test is called uniformly consistent, if for any $\varepsilon>0$ there is a sample size $n$ such that the probability of error on a sample of

*INRIA Lille-Nord Europe, 40, avenue Halley, 59650 Villeneuve d'Ascq, France, daniil@ryabko.net 
size larger than $n$ is not greater than $\varepsilon$ if any distribution from $H_{0} \cup H_{1}$ is chosen to generate the sample. Thus, a uniformly consistent test provides performance guarantees for finite sample sizes.

The results. Here we obtain some topological conditions of the hypotheses for which consistent tests exist, for the case of stationary ergodic distributions.

A distributional distance between two process distributions [3] is defined as a weighted sum of probabilities of all possible tuples $X \in A^{*}$, where $A$ is the alphabet and the weights are positive and have a finite sum.

The test $\varphi_{H_{0}, H_{1}}$ that we construct is based on empirical estimates of distributional distance. It outputs 0 if the given sample is closer to the (closure of) $H_{0}$ than to the (closure of) $H_{1}$, and outputs 1 otherwise. The main result is as follows.

Theorem. Let $H_{0}, H_{1} \subset \mathcal{E}$, where $\mathcal{E}$ is the set of all stationary ergodic process distributions. If, for each $i \in\{0,1\}$ the set $H_{i}$ has probability 1 with respect to ergodic decompositions of every element of $H_{i}$, then there is a uniformly consistent test for $H_{0}$ against $H_{1}$. Conversely, if there is a uniformly consistent test for $H_{0}$ against $H_{1}$, then, for each $i \in\{0,1\}$, the set $H_{1-i}$ has probability 0 with respect to ergodic decompositions of every element of $H_{i}$.

Prior work. This work continuous our previous research [13, 14], which provides similar necessary and sufficient conditions for the existence of a consistent test, for a weaker notion of asymmetric consistency: Type I error is uniformly bounded, while Type II error is required to tend to 0 as the sample size grows.

Besides that, there is of course a vast body of literature on hypothesis testing for i.i.d. (real- or discrete-valued) data (see e.g. [7, 4]). There is, however, much less literature on hypothesis testing beyond i.i.d. or parametric models. For a weaker notion of consistency, namely, requiring that the test should stabilize on the correct answer for a.e. realization of the process (under either $H_{0}$ or $H_{1}$ ), [6] constructs a consistent test for so-called constrained finite-state model classes (including finite-state Markov and hidden Markov processes), against the general alternative of stationary ergodic processes. For the same notion of consistency, [8] gives sufficient conditions on two hypotheses $H_{0}$ and $H_{1}$ that consist of stationary ergodic real-valued processes, under which a consistent test exists, extending the results of [2] for i.i.d. data. The latter condition is that $H_{0}$ and $H_{1}$ are contained in disjoint $F_{\sigma}$ sets (countable unions of closed sets), with respect to the topology of weak convergence. Asymmetrically consistent tests for some specific hypotheses, but under the general alternative of stationary ergodic processes, have been proposed in $[9,10,15,16]$, which address problems of testing identity, independence, estimating the order of a Markov process, and also the change point problem. Noteworthy, a conceptually simple hypothesis of homogeneity (testing whether two sample are generated by the same or by different processes) does not admit a consistent test even in the weakest asymptotic sense, as was shown in [12]. Empirical estimates of distributional distance have been also used to address the problem of clustering time series $[11,5]$. 


\section{Preliminaries}

Let $A$ be a finite alphabet, and denote $A^{*}$ the set of words (or tuples) $\cup_{i=1}^{\infty} A^{i}$. For a word $B$ the symbol $|B|$ stands for the length of $B$. Denote $B_{i}$ the $i$ th element of $A^{*}$, enumerated in such a way that the elements of $A^{i}$ appear before the elements of $A^{i+1}$, for all $i \in \mathbb{N}$. Distributions or (stochastic) processes are probability measures on the space $\left(A^{\infty}, \mathcal{F}_{A^{\infty}}\right)$, where $\mathcal{F}_{A^{\infty}}$ is the Borel sigmaalgebra of $A^{\infty}$. Denote $\#(X, B)$ the number of occurrences of a word $B$ in a word $X \in A^{*}$ and $\nu(X, B)$ its frequency: $\#(X, B)=\sum_{i=1}^{|X|-|B|+1} I_{\left\{\left(X_{i}, \ldots, X_{i+|B|-1}\right)=B\right\}}$, and

$$
\nu(X, B)=\left\{\begin{array}{cc}
\frac{1}{|X|-|B|+1} \#(X, B) & \text { if }|X| \geq|B|, \\
0 & \text { otherwise }
\end{array}\right.
$$

where $X=\left(X_{1}, \ldots, X_{|X|}\right)$. For example, $\nu(0001,00)=2 / 3$.

We use the abbreviation $X_{1 . . k}$ for $X_{1}, \ldots, X_{k}$. A process $\rho$ is stationary if

$$
\rho\left(X_{1 . .|B|}=B\right)=\rho\left(X_{t . . t+|B|-1}=B\right)
$$

for any $B \in A^{*}$ and $t \in \mathbb{N}$. Denote $\mathcal{S}$ the set of all stationary processes on $A^{\infty}$. A stationary process $\rho$ is called (stationary) ergodic if the frequency of occurrence of each word $B$ in a sequence $X_{1}, X_{2}, \ldots$ generated by $\rho$ tends to its a priori (or limiting) probability a.s.: $\rho\left(\lim _{n \rightarrow \infty} \nu\left(X_{1 . . n}, B\right)=\rho\left(X_{1 . .|B|}=B\right)\right)=1$. Denote $\mathcal{E}$ the set of all stationary ergodic processes.

A distributional distance is defined for a pair of processes $\rho_{1}, \rho_{2}$ as follows [3]:

$$
d\left(\rho_{1}, \rho_{2}\right)=\sum_{i=1}^{\infty} w_{i}\left|\rho_{1}\left(X_{1 . .\left|B_{i}\right|}=B_{i}\right)-\rho_{2}\left(X_{1 . .\left|B_{i}\right|}=B_{i}\right)\right|
$$

where $w_{i}$ are summable positive real weights (e.g. $w_{k}=2^{-k}$ : we fix this choice for the sake of concreteness). It is easy to see that $d$ is a metric. Equipped with this metric, the space of all stochastic processes is a compact, and the set of stationary processes $\mathcal{S}$ is its convex closed subset. (The set $\mathcal{E}$ is not closed.) When talking about closed and open subsets of $\mathcal{S}$ we assume the topology of $d$. Compactness of the set $\mathcal{S}$ is one of the main ingredients in the proofs of the main results. Another is that the distance $d$ can be consistently estimated, as the following lemma shows (because of its importance for further development, we give it with a proof).

Lemma 1 ( $\hat{d}$ is consistent $[15,16])$. Let $\rho, \xi \in \mathcal{E}$ and let a sample $X_{1 . . k}$ be generated by $\rho$. Then

$$
\lim _{k \rightarrow \infty} \hat{d}\left(X_{1 . . k}, \xi\right)=d(\rho, \xi) \rho \text {-a.s. }
$$

Proof. For any $\varepsilon>0$ find such an index $J$ that $\sum_{i=J}^{\infty} w_{i}<\varepsilon / 2$. For each $j$ we have $\lim _{k \rightarrow \infty} \nu\left(X_{1 . . k}, B_{j}\right)=\rho\left(B_{j}\right)$ a.s., so that $\left|\nu\left(X_{1 . . k}, B_{j}\right)-\rho\left(B_{j}\right)\right|<\varepsilon /\left(2 J w_{j}\right)$ 
from some $k$ on; denote $K_{j}$ this $k$. Let $K=\max _{j<J} K_{j}$ ( $K$ depends on the realization $\left.X_{1}, X_{2}, \ldots\right)$. Thus, for $k>K$ we have

$$
\begin{array}{r}
\left|\hat{d}\left(X_{1 . . k}, \xi\right)-d(\rho, \xi)\right|=\left|\sum_{i=1}^{\infty} w_{i}\left(\left|\nu\left(X_{1 . . k}, B_{i}\right)-\xi\left(B_{i}\right)\right|-\left|\rho\left(B_{i}\right)-\xi\left(B_{i}\right)\right|\right)\right| \\
\leq \sum_{i=1}^{\infty} w_{i}\left|\nu\left(X_{1 . . k}, B_{i}\right)-\rho\left(B_{i}\right)\right| \leq \sum_{i=1}^{J} w_{i}\left|\nu\left(X_{1 . . k}, B_{i}\right)-\rho_{X}\left(B_{i}\right)\right|+\varepsilon / 2 \\
\leq \sum_{i=1}^{J} w_{i} \varepsilon /\left(2 J w_{i}\right)+\varepsilon / 2=\varepsilon
\end{array}
$$

which proves the statement.

Considering the Borel (with respect to the metric $d$ ) sigma-algebra $\mathcal{F}_{\mathcal{S}}$ on the set $\mathcal{S}$, we obtain a standard probability space $\left(\mathcal{S}, \mathcal{F}_{\mathcal{S}}\right)$. An important tool that will be used in the analysis is ergodic decomposition of stationary processes (see e.g. $[3,1]$ ): any stationary process can be expressed as a mixture of stationary ergodic processes. More formally, for any $\rho \in \mathcal{S}$ there is a measure $W_{\rho}$ on $\left(\mathcal{S}, \mathcal{F}_{\mathcal{S}}\right)$, such that $W_{\rho}(\mathcal{E})=1$, and $\rho(B)=\int d W_{\rho}(\mu) \mu(B)$, for any $B \in \mathcal{F}_{A^{\infty}}$. The support of a stationary distribution $\rho$ is the minimal closed set $U \subset \mathcal{S}$ such that $W_{\rho}(U)=1$.

A test is a function $\varphi: A^{*} \rightarrow\{0,1\}$ that takes a sample and outputs a binary answer, where the answer $i$ is interpreted as "the sample was generated by a distribution that belongs to $H_{i}$ ". The answer $i$ is correct if the sample was indeed generated by a distribution from $H_{i}$, otherwise we say that the test made an error.

A test $\varphi$ is called uniformly consistent if for every $\alpha$ there is an $n_{\alpha} \in \mathbb{N}$ such that for every $n \geq n_{\alpha}$ the probability of error on a sample of size $n$ is less than $\alpha: \rho\left(X \in A^{n}: \varphi(X)=i\right)<\alpha$ for every $\rho \in H_{1-i}$ and every $i \in\{0,1\}$.

\section{Main results}

The tests presented below are based on empirical estimates of the distributional distance $d$ :

$$
\hat{d}\left(X_{1 . . n}, \rho\right)=\sum_{i=1}^{\infty} w_{i}\left|\nu\left(X_{1 . . n}, B_{i}\right)-\rho\left(B_{i}\right)\right|,
$$

where $n \in \mathbb{N}, \rho \in \mathcal{S}, X_{1 . . n} \in A^{n}$. That is, $\hat{d}\left(X_{1 . . n}, \rho\right)$ measures the discrepancy between empirically estimated and theoretical probabilities. For a sample $X_{1 . . n} \in A^{n}$ and a hypothesis $H \subset \mathcal{E}$ define

$$
\hat{d}\left(X_{1 . . n}, H\right)=\inf _{\rho \in H} \hat{d}\left(X_{1 . . n}, \rho\right) .
$$

For $H \subset \mathcal{S}$, denote $\mathrm{cl} H$ the closure of $H$ (with respect to the topology of $d$ ). 
For $H_{0}, H_{1} \subset \mathcal{S}$, the uniform test $\varphi_{H_{0}, H_{1}}$ is constructed as follows. For each $n \in \mathbb{N}$ let

$$
\varphi_{H_{0}, H_{1}}\left(X_{1 . . n}\right):= \begin{cases}0 & \text { if } \hat{d}\left(X_{1 . . n}, \operatorname{cl} H_{0} \cap \mathcal{E}\right)<\hat{d}\left(X_{1 . . n}, \operatorname{cl} H_{1} \cap \mathcal{E}\right), \\ 1 & \text { otherwise. }\end{cases}
$$

Theorem 1 (uniform testing). Let $H_{0} \subset \mathcal{S}$ and $H_{1} \subset \mathcal{S}$. If $W_{\rho}\left(H_{i}\right)=1$ for every $\rho \in \mathrm{cl} H_{i}$ then the test $\varphi_{H_{0}, H_{1}}$ is uniformly consistent. Conversely, if there exists a uniformly consistent test for $H_{0}$ against $H_{1}$ then $W_{\rho}\left(H_{1-i}\right)=0$ for any $\rho \in \mathrm{clH}_{i}$.

The proof is deferred to section 5 .

\section{Examples}

First of all, it is obvious that sets that consist of just one or finitely many stationary ergodic processes are closed and closed under ergodic decompositions; therefore, for any pair of disjoint sets of this type, there exists a uniformly consistent test. (In particular, there is a uniformly consistent test for $H_{0}=\left\{\rho_{0}\right\}$ against $H_{1}=\left\{\rho_{1}\right\}$, where $\rho_{0}, \rho_{1} \in \mathcal{E}$.)

It is clear that for any $\rho_{0}$ there is no uniformly consistent test for $\left\{\rho_{0}\right\}$ against $\mathcal{E} \backslash\left\{\rho_{0}\right\}$. More generally, for any non-empty $H_{0}$ there is no uniformly consistent test for $H_{0}$ against $\mathcal{E} \backslash H_{0}$ provided the latter complement is also non-empty. Indeed, this follows from Theorem 1 since in these cases the closures of $H_{0}$ and $H_{1}$ are not disjoint. One might suggest at this point that a uniformly consistent test exists if we restrict $H_{1}$ to those processes that are sufficiently far from $\rho_{0}$. However, this is not true. We can prove an even stronger negative result.

Proposition 1. Let $\rho, \nu \in \mathcal{E}, \rho \neq \nu$ and let $\varepsilon>0$. There is no uniformly consistent test for $H_{0}=\{\rho\}$ against $H_{1}=\left\{\nu^{\prime} \in \mathcal{E}: d\left(\nu^{\prime}, \nu\right) \leq \varepsilon\right\}$.

The proof of the proposition is deferred to the next section. What the proposition means is that, while distributional distance is well suited for characterizing those hypotheses for which consistent test exist, it is not suited for formulating the actual hypotheses. Apparently a stronger distance is needed for the latter.

The following statement is easy to demonstrate from Theorem 1.

Corollary 1. Given two disjoint sets $H_{0}$ and $H_{1}$ each of which is continuously parametrized by a compact set of parameters and is closed under taking ergodic decompositions, there exists a uniformly consistent test of $H_{0}$ against $H_{1}$.

Examples of parametrisations mentioned in the Corollary are the sets of $k$ order Markov sources, parametrised by transition probabilities. Thus, any two disjoint closed subsets of these sets satisfy the assumption of the Corollary. 


\section{Proofs}

The proof of Theorem 1 will use the following lemmas, whose proofs can be found in [14].

Lemma 2 (smooth probabilities of deviation). Let $m>2 k>1, \rho \in \mathcal{S}, H \subset \mathcal{S}$, and $\varepsilon>0$. Then

$$
\rho\left(\hat{d}\left(X_{1 . . m}, H\right) \geq \varepsilon\right) \leq 2 \varepsilon^{\prime-1} \rho\left(\hat{d}\left(X_{1 . . k}, H\right) \geq \varepsilon^{\prime}\right),
$$

where $\varepsilon^{\prime}:=\varepsilon-\frac{2 k}{m-k+1}-t_{k}$ with $t_{k}$ being the sum of all the weights of tuples longer than $k$ in the definition of $d: t_{k}:=\sum_{i:\left|B_{i}\right|>k} w_{i}$. Further,

$$
\rho\left(\hat{d}\left(X_{1 . . m}, H\right) \leq \varepsilon\right) \leq 2 \rho\left(\hat{d}\left(X_{1 . . k}, H\right) \leq \frac{m}{m-k+1} 2 \varepsilon+\frac{4 k}{m-k+1}\right) .
$$

The meaning of this lemma is as follows. For any word $X_{1 . . m}$, if it is far away from (or close to) a given distribution $\mu$ (in the empirical distributional distance), then some of its shorter subwords $X_{i . i+k}$ are far from (close to) $\mu$ too. In other words, for a stationary distribution $\mu$, it cannot happen that a small sample is likely to be close to $\mu$, but a larger sample is likely to be far.

Lemma 3. Let $\rho_{k} \in \mathcal{S}, k \in \mathbb{N}$ be a sequence of processes that converges to a process $\rho_{*}$. Then, for any $T \in A^{*}$ and $\varepsilon>0$ if $\rho_{k}(T)>\varepsilon$ for infinitely many indices $k$, then $\rho_{*}(T) \geq \varepsilon$.

This statement follows from the fact that $\rho(T)$ is continuous as a function of $\rho$.

Proof of Theorem 1. To prove the first statement of the theorem, we will show that the test $\varphi_{H_{0}, H_{1}}$ is a uniformly consistent test for $\mathrm{cl} H_{0} \cap \mathcal{E}$ against cl $H_{1} \cap \mathcal{E}$ (and hence for $H_{0}$ against $H_{1}$ ), under the conditions of the theorem. Suppose that, on the contrary, for some $\alpha>0$ for every $n^{\prime} \in \mathbb{N}$ there is a process $\rho \in \operatorname{cl} H_{0}$ such that $\rho\left(\varphi\left(X_{1 . . n}\right)=1\right)>\alpha$ for some $n>n^{\prime}$. Define

$$
\Delta:=d\left(\operatorname{cl} H_{0}, \operatorname{cl} H_{1}\right):=\inf _{\rho_{0} \in \operatorname{cl} H_{0} \cap \mathcal{E}, \rho_{1} \in \operatorname{cl} H_{1} \cap \mathcal{E}} d\left(\rho_{0}, \rho_{1}\right),
$$

which is positive since $\mathrm{cl} H_{0}$ and $\mathrm{cl} H_{1}$ are closed and disjoint. We have

$$
\begin{aligned}
& \alpha<\rho\left(\varphi\left(X_{1 . . n}\right)=1\right) \\
& \leq \rho\left(\hat{d}\left(X_{1 . . n}, H_{0}\right) \geq \Delta / 2 \text { or } \hat{d}\left(X_{1 . . n}, H_{1}\right)<\Delta / 2\right) \\
& \quad \leq \rho\left(\hat{d}\left(X_{1 . . n}, H_{0}\right) \geq \Delta / 2\right)+\rho\left(\hat{d}\left(X_{1 . . n}, H_{1}\right)<\Delta / 2\right) .
\end{aligned}
$$

This implies that either $\rho\left(\hat{d}\left(X_{1 . . n}, \operatorname{cl} H_{0}\right) \geq \Delta / 2\right)>\alpha / 2$ or $\rho\left(\hat{d}\left(X_{1 . . n}, \operatorname{cl} H_{1}\right)<\right.$ $\Delta / 2)>\alpha / 2$, so that, by assumption, at least one of these inequalities holds for infinitely many $n \in \mathbb{N}$ for some sequence $\rho_{n} \in H_{0}$. Suppose that it is the first one, that is, there is an increasing sequence $n_{i}, i \in \mathbb{N}$ and a sequence $\rho_{i} \in \operatorname{cl} H_{0}$, $i \in \mathbb{N}$ such that

$$
\rho_{i}\left(\hat{d}\left(X_{1 . . n_{i}}, \operatorname{cl} H_{0}\right) \geq \Delta / 2\right)>\alpha / 2 \text { for all } i \in \mathbb{N} .
$$


The set $\mathcal{S}$ is compact, hence so is its closed subset cl $H_{0}$. Therefore, the sequence $\rho_{i}, i \in \mathbb{N}$ must contain a subsequence that converges to a certain process $\rho_{*} \in$ $\mathrm{cl} H_{0}$. Passing to a subsequence if necessary, we may assume that this convergent subsequence is the sequence $\rho_{i}, i \in \mathbb{N}$ itself.

Using Lemma 2, (3) (with $\rho=\rho_{n_{m}}, m=n_{m}, k=n_{k}$, and $H=\mathrm{cl} H_{0}$ ), and taking $k$ large enough to have $t_{n_{k}}<\Delta / 4$, for every $m$ large enough to have $\frac{2 n_{k}}{n_{m}-n_{k}+1}<\Delta / 4$, we obtain

$$
8 \Delta^{-1} \rho_{n_{m}}\left(\hat{d}\left(X_{1 . . n_{k}}, \operatorname{cl} H_{0}\right) \geq \Delta / 4\right) \geq \rho_{n_{m}}\left(\hat{d}\left(X_{1 . . n_{m}}, \operatorname{cl} H_{0}\right) \geq \Delta / 2\right)>\alpha / 2 .
$$

That is, we have shown that for any large enough index $n_{k}$ the inequality $\rho_{n_{m}}\left(\hat{d}\left(X_{1 . . n_{k}}, \operatorname{cl} H_{0}\right) \geq \Delta / 4\right)>\Delta \alpha / 16$ holds for infinitely many indices $n_{m}$. From this and Lemma 3 with $T=T_{k}:=\left\{X: \hat{d}\left(X_{1 . . n_{k}}, \operatorname{cl} H_{0}\right) \geq \Delta / 4\right\}$ we conclude that $\rho_{*}\left(T_{k}\right)>\Delta \alpha / 16$. The latter holds for infinitely many $k$; that is, $\rho_{*}\left(\hat{d}\left(X_{1 . . n_{k}}, \operatorname{cl} H_{0}\right) \geq \Delta / 4\right)>\Delta \alpha / 16$ infinitely often. Therefore,

$$
\rho_{*}\left(\limsup _{n \rightarrow \infty} d\left(X_{1 . . n}, \operatorname{cl} H_{0}\right) \geq \Delta / 4\right)>0 .
$$

However, we must have

$$
\rho_{*}\left(\lim _{n \rightarrow \infty} d\left(X_{1 . . n}, \operatorname{cl} H_{0}\right)=0\right)=1
$$

for every $\rho_{*} \in \operatorname{cl} H_{0}$ : indeed, for $\rho_{*} \in \operatorname{cl} H_{0} \cap \mathcal{E}$ it follows from Lemma 1, and for $\rho_{*} \in \mathrm{cl} H_{0} \backslash \mathcal{E}$ from Lemma 1, ergodic decomposition and the conditions of the theorem.

Thus, we have arrived at a contradiction that shows that $\rho_{n}\left(\hat{d}\left(X_{1 . . n}, \operatorname{cl} H_{0}\right)>\right.$ $\Delta / 2)>\alpha / 2$ cannot hold for infinitely many $n \in \mathbb{N}$ for any sequence of $\rho_{n} \in$ cl $H_{0}$. Analogously, we can show that $\rho_{n}\left(\hat{d}\left(X_{1 . . n}, \operatorname{cl} H_{1}\right)<\Delta / 2\right)>\alpha / 2$ cannot hold for infinitely many $n \in \mathbb{N}$ for any sequence of $\rho_{n} \in \mathrm{cl} H_{0}$. Indeed, using Lemma 2, equation (4), we can show that $\rho_{n_{m}}\left(\hat{d}\left(X_{1 . . n_{m}}, \operatorname{cl} H_{1}\right) \leq \Delta / 2\right)>\alpha / 2$ for a large enough $n_{m}$ implies $\rho_{n_{m}}\left(\hat{d}\left(X_{1 . . n_{k}}, \mathrm{cl} H_{1}\right) \leq 3 \Delta / 4\right)>\alpha / 4$ for a smaller $n_{k}$. Therefore, if we assume that $\rho_{n}\left(\hat{d}\left(X_{1 . . n}, \operatorname{cl} H_{1}\right)<\Delta / 2\right)>\alpha / 4$ for infinitely many $n \in \mathbb{N}$ for some sequence of $\rho_{n} \in \operatorname{cl} H_{0}$, then we will also find a $\rho_{*}$ for which $\rho_{*}\left(\hat{d}\left(X_{1 . . n}, \operatorname{cl} H_{1}\right) \leq 3 \Delta / 4\right)>\alpha / 4$ for infinitely many $n$, which, using Lemma 1 and ergodic decomposition, can be shown to contradict the fact that $\rho_{*}\left(\lim _{n \rightarrow \infty} d\left(X_{1 . . n}, \operatorname{cl} H_{1}\right) \geq \Delta\right)=1$.

Thus, returning to (5), we have shown that from some $n$ on there is no $\rho \in \operatorname{cl} H_{0}$ for which $\rho(\varphi=1)>\alpha$ holds true. The statement for $\rho \in \operatorname{cl} H_{1}$ can be proven analogously, thereby finishing the proof of the first statement.

To prove the second statement of the theorem, we assume that there exists a uniformly consistent test $\varphi$ for $H_{0}$ against $H_{1}$, and we will show that $W_{\rho}\left(H_{1-i}\right)=0$ for every $\rho \in \operatorname{cl} H_{i}$. Indeed, let $\rho \in \operatorname{cl} H_{0}$, that is, suppose that there is a sequence $\xi_{i} \in H_{0}, i \in \mathbb{N}$ such that $\xi_{i} \rightarrow \rho$. Assume $W_{\rho}\left(H_{1}\right)=\delta>0$ and take $\alpha:=\delta / 2$. Since the test $\varphi$ is uniformly consistent, there is an $N \in \mathbb{N}$ 
such that for every $n>N$ we have

$$
\begin{aligned}
\rho\left(\varphi\left(X_{1 . . n}=0\right)\right) \leq \int_{H_{1}} \varphi\left(X_{1 . . n}=0\right) d W_{\rho}+\int_{\mathcal{E} \backslash H_{1}} \varphi\left(X_{1 . . n}=0\right) d W_{\rho} \\
\leq \delta \alpha+1-\delta \leq 1-\delta / 2 .
\end{aligned}
$$

Recall that, for $T \in A^{*}, \mu(T)$ is a continuous function in $\mu$. In particular, this holds for the set $T=\left\{X \in A^{n}: \varphi(X)=0\right\}$, for any given $n \in \mathbb{N}$. Therefore, for every $n>N$ and for every $i$ large enough, $\rho_{i}\left(\varphi\left(X_{1 . . n}\right)=0\right)<1-\delta / 2$ implies also $\xi_{i}\left(\varphi\left(X_{1 . . n}\right)=0\right)<1-\delta / 2$ which contradicts $\xi_{i} \in H_{0}$. This contradiction shows $W_{\rho}\left(H_{1}\right)=0$ for every $\rho \in \operatorname{cl} H_{0}$. The case $\rho \in \operatorname{cl} H_{1}$ is analogous. The theorem is proven.

Proof of Proposition 1. Assume $d(\rho, \nu)>\varepsilon$ (the other case is obvious). Consider the process $\left(x_{1}, y_{1}\right),\left(x_{2}, y_{2}\right), \ldots$ on pairs $\left(x_{i}, y_{i}\right) \in A^{2}$, such that the distribution of $x_{1}, x_{2}, \ldots$ is $\nu$, the distribution of $y_{1}, y_{2}, \ldots$ is $\rho$ and the two components $x_{i}$ and $y_{i}$ are independent; in other words, the distribution of $\left(x_{i}, y_{i}\right)$ is $\nu \times \rho$. Consider also a two-state stationary ergodic Markov chain $\mu$, with two states 1 and 2 , whose transition probabilities are $\left(\begin{array}{cc}1-p & p \\ q & 1-q\end{array}\right)$, where $0<p<q<1$. The limiting (and initial) probability of the state 1 is $p /(p+q)$ and that of the state 2 is $q /(p+q)$. Finally, the process $z_{1}, z_{2}, \ldots$ is constructed as follows: $z_{i}=x_{i}$ if $\mu$ is in the state $a$ and $z_{i}=y_{i}$ otherwise (here it is assumed that the chain $\mu$ generates a sequence of outcomes independently of $\left(x_{i}, y_{i}\right)$. Clearly, for every $p, q$ satisfying $0<p<q<1$ the process $z_{1}, z_{2}, \ldots$ is stationary ergodic; denote $\zeta$ its distribution. Let $p_{n}:=1 /(n+1), n \in \mathbb{N}$. Since $d(\rho, \nu)>\varepsilon$, we can find a $\delta>0$ such that $d\left(\rho, \zeta_{n}\right)>\varepsilon$ where $\zeta_{n}$ is the distribution $\zeta$ with parameters $p_{n}$ and $q_{n}$, where $q_{n}$ satisfies $q_{n} /\left(p_{n}+q_{n}\right)=\delta$. Thus, $\zeta_{n} \in H_{1}$ for all $n \in \mathbb{N}$. However, $\lim _{n \rightarrow \infty} \zeta_{n}=\zeta_{\infty}$ where $\zeta_{\infty}$ is the stationary distribution with $W_{\zeta_{\infty}}(\rho)=\delta$ and $W_{\zeta_{\infty}}(\nu)=1-\delta$. Therefore, $\zeta_{\infty} \in \operatorname{cl} H_{1}$ and $W_{\zeta_{\infty}}\left(H_{0}\right)>0$, so that by Theorem 1 there is no uniformly consistent test for $H_{0}$ against $H_{1}$, which concludes the proof.

\section{Acknowledgements}

This work has been partially supported by the French Ministry of Higher Education and Research, Nord-Pas de Calais Regional Council and FEDER through the "Contrat de Projets Etat Region (CPER) 2007-2013."

\section{References}

[1] P. Billingsley. Ergodic theory and information. Wiley, New York, 1965.

[2] A. Dembo and Y. Peres. A topological criterion for hypothesis testing. Ann. Math. Stat., 22:106-117, 1994. 
[3] R. Gray. Probability, Random Processes, and Ergodic Properties. Springer Verlag, 1988.

[4] M.G. Kendall and A. Stuart. The advanced theory of statistics; Vol.2: Inference and relationship. London, 1961.

[5] A. Khaleghi, D. Ryabko, J. Mary, and P. Preux. Online clustering of processes. In AISTATS, JMLR W\&CP 22, pages 601-609, 2012.

[6] J.C. Kieffer. Strongly consistent code-based identification and order estimation for constrained finite-state model classes. IEEE Transactions on Information Theory, 39(3):893-902, 1993.

[7] E. Lehmann. Testing Statistical Hypotheses, 2nd edition. Wiley, New York, 1986.

[8] A. Nobel. Hypothesis testing for families of ergodic processes. Bernoulli, 12(2):251-269, 2006.

[9] B. Ryabko and J. Astola. Universal codes as a basis for time series testing. Statistical Methodology, 3:375-397, 2006.

[10] B. Ryabko, J. Astola, and A. Gammerman. Application of Kolmogorov complexity and universal codes to identity testing and nonparametric testing of serial independence for time series. Theoretical Computer Science, 359:440-448, 2006.

[11] D. Ryabko. Clustering processes. In Proc. the 27th International Conference on Machine Learning (ICML 2010), pages 919-926, Haifa, Israel, 2010.

[12] D. Ryabko. Discrimination between B-processes is impossible. Journal of Theoretical Probability, 23(2):565-575, 2010.

[13] D. Ryabko. Testing composite hypotheses about discrete-valued stationary processes. In Proc. IEEE Information Theory Workshop (ITW'10), pages 291-295, Cairo, Egypt, 2010. IEEE.

[14] D. Ryabko. Testing composite hypotheses about discrete ergodic processes. Test, 21(2):317-329, 2012.

[15] D. Ryabko and B. Ryabko. On hypotheses testing for ergodic processes. In Proc. 2008 IEEE Information Theory Workshop, pages 281-283, Porto, Portugal, 2008. IEEE.

[16] D. Ryabko and B. Ryabko. Nonparametric statistical inference for ergodic processes. IEEE Transactions on Information Theory, 56(3):1430-1435, 2010 . 